\title{
Application of an Optimal Control Allocation Scheme with Structural Load and Aero Heating Feedback for a Morphing Inflatable Aerodynamic Decelerator
}

\author{
George Z. Bassett* \\ University of Texas at Austin, Austin, Texas, 78712 \\ Jing Pei ${ }^{\dagger}$, Adam Milne Slagle ${ }^{\ddagger}$, and Bandu Pamadi $§$ \\ NASA Langley Research Center, Hampton, Virginia, 23681
}

\begin{abstract}
Precision landing of large payloads on Mars presents a challenge to the Entry, Descent, and Landing (EDL) community. Previous studies indicated that by incorporating the capability for a Hypersonic Inflatable Aerodynamic Decelerator (HIAD) to morph during reentry would result in a more accurate landing footprint by allowing modulation of the liftto-drag (L/D) vector directly instead of through bank angle control. However, morphing the HIAD shape for trajectory control may expose the HIAD to potential structural loads or aero heating concerns. In this study, the application of an optimal control allocation (OCA) technique was investigated that would to enable the morphing HIAD to maximize trajectory control capabilities while simultaneously keeping the structural loads and aero heating below some thresholds. This concept was demonstrated in a 3 degree-of-freedom (DOF) EDL simulation and provides basis for future research.
\end{abstract}

\section{Nomenclature}

$C_{L} \quad$ Coefficient of Lift

$C_{D} \quad$ Coefficient of Drag

$\alpha \quad$ Angle of Attack, degrees

$\beta \quad$ Sideslip Angle, degrees

$C_{F x} \quad$ Coefficient of Force, x-direction

$C_{F y} \quad$ Coefficient of Force, y-direction

$C_{F z} \quad$ Coefficient of Force, z-direction

$B \quad$ Control Effectiveness Matrix

$u \quad$ Deflection of Cable

$l \quad$ Structural Load on Cable

$\dot{q} \quad$ Heat Rate

$U_{\infty} \quad$ Free-stream Velocity

$\rho \quad$ Free-stream Density

$r_{\text {nose }} \quad$ Nose Radius

$a_{d} \quad$ Control Command

$\varepsilon \quad$ Trim Cost Weight

$H \quad$ Trim Tuning Matrix

$\gamma \quad$ Structural Load Constraint Weight

$M \quad$ Measured Structural Loads Vector

$\delta \quad$ Cable Deflection, meters

\footnotetext{
${ }^{*}$ Currently: Graduate Student, Georgia Institute of Technology

${ }^{\dagger}$ Aerospace Engineer, Vehicle Analysis Branch

${ }^{\ddagger}$ Pathways Student, Vehicle Analysis Branch

${ }^{\S}$ Retired Aerospace Engineer, Vehicle Analysis Branch
} 


$\begin{array}{ll}L & \text { Matrix of Surface Influence Coefficients for Each Measured Structural Load } \\ n & \text { Structural Load Constraint Steepness } \\ \psi & \text { Heat Flux Load Constraint Weight } \\ Q & \text { Measured Heat Flux Loads Vector } \\ P & \text { Matrix of Surface Influence Coefficients for Each Measured Heat Flux Load } \\ m & \text { Heat Flux Load Constraint Steepness } \\ R & \text { Relaxation Factor } \\ \dagger & \text { Moore Penrose Pseudoinverse } \\ N & \text { Structural Load Cost Function Simplification Parameter } \\ F & \text { Heat Flux Load Cost Function Simplification Parameter } \\ \gamma & \text { Flight Path Angle } \\ R & \text { Flight Range from Present Position to Target } \\ L / D & \text { Lift to Drag Ratio } \\ D / W & \text { Non-dimensional Drag Acceleration } \\ K_{1} & \text { Variable Gain, Flight Path Angle Term } \\ K_{2} & \text { Variable Gain, Drag to Weight Term } \\ K_{3} & \text { Variable Gain, Range Term } \\ v_{e} & \text { Entry Velocity } \\ h_{o} & \text { Entry Altitude } \\ & \\ \text { Subscript } & \\ n & \text { Cable or Control Surface Number } \\ k & \text { Iteration Number } \\ r e f & \text { State Variables at Reference Trajectory }\end{array}$

\section{Introduction}

Conventional rigid aeroshells used for reentry are coming to the edge of their operational limits in terms of allowable mass and size. The largest entry vehicle demonstrated on Mars had an aeroshell diameter of $4.5 \mathrm{~m}$ and delivered a $950 \mathrm{~kg}$ payload. ${ }^{1}$ The purpose of a Hypersonic Inflatable Aerodynamic Decelerator (HIAD) is to overcome many of the operational constraints (packaging, scalability, etc) associated with a rigid aeroshell. ${ }^{1}$ In July 2012, NASA successfully demonstrated the Inflatable Reentry Vehicle Experiment $\left(\right.$ IRVE3) ${ }^{2}$ to investigate the survivability of the Flexible Thermal Protection System (FTPS) to heating rates encountered during reentry and notionally demonstrate the effectiveness of using Center of Gravity Offset (CGO) subsystem for altering the vehicle's lift to drag (L/D) ratio.

In addition to many of the operational advantages, the morphing HIAD concept allows for potential active control of L/D via shape manipulation and is considered a form of direct force control (DFC). Reference 3 shows the potential benefits of DFC compared to the current state-of-the-art. DFC can significantly increase the landing footprint accuracy and reduce the total required propellant mass. Furthermore, conventional approach of jettison ballast to adjust the center of mass location to achieve the desired L/D ratio may not be feasible with large entry masses associated with human scale missions. Work by Green ${ }^{1}$ used a combination of engineering and analytical methods to evaluate the aerodynamics of various morphed aeroshell configurations. Furthermore, it was demonstrated that the morphed aeroshell shape can be optimized to maximize L/D while keeping the stagnation point heating throughout the reentry trajectory below a certain threshold. Green's work evaluated the trajectory impact of a discrete morphing event in a 3 DOF simulation but did not analyze the continuous morphing scenario.

Figure 1 shows the un-morphed HIAD configuration in the top row. The second and third rows depict various morphed shapes with the bottom section deformed or "deflected" at different magnitudes. At $\theta=$ 0 , the upper-center portion of the aeroshell would encounter the greatest amount of morphing while the deformation would taper off to zero at $\pm \theta_{\max }$. The idea is to deform the upper and lower portions the aeroshell for pitch control while the left and right sections for yaw control. These sections would morph throughout the reentry phase and provide continuous corrections to down range and cross range errors. Doing so would improve the maneuverability and landing accuracy of the HIAD compare to conventional bank angle control. ${ }^{3}$ A separate effort by Slagle ${ }^{4}$ was performed in assessing the practicability of a continuous 
morphing HIAD from a structural and mechanical perspective. The study considered using cables (that could extend and retract) to morph portions of the aeroshell. Figure 2 shows a mock up of the HIAD/cable concept. The loads on the cables is proportional to the amount of desired surface deformation.
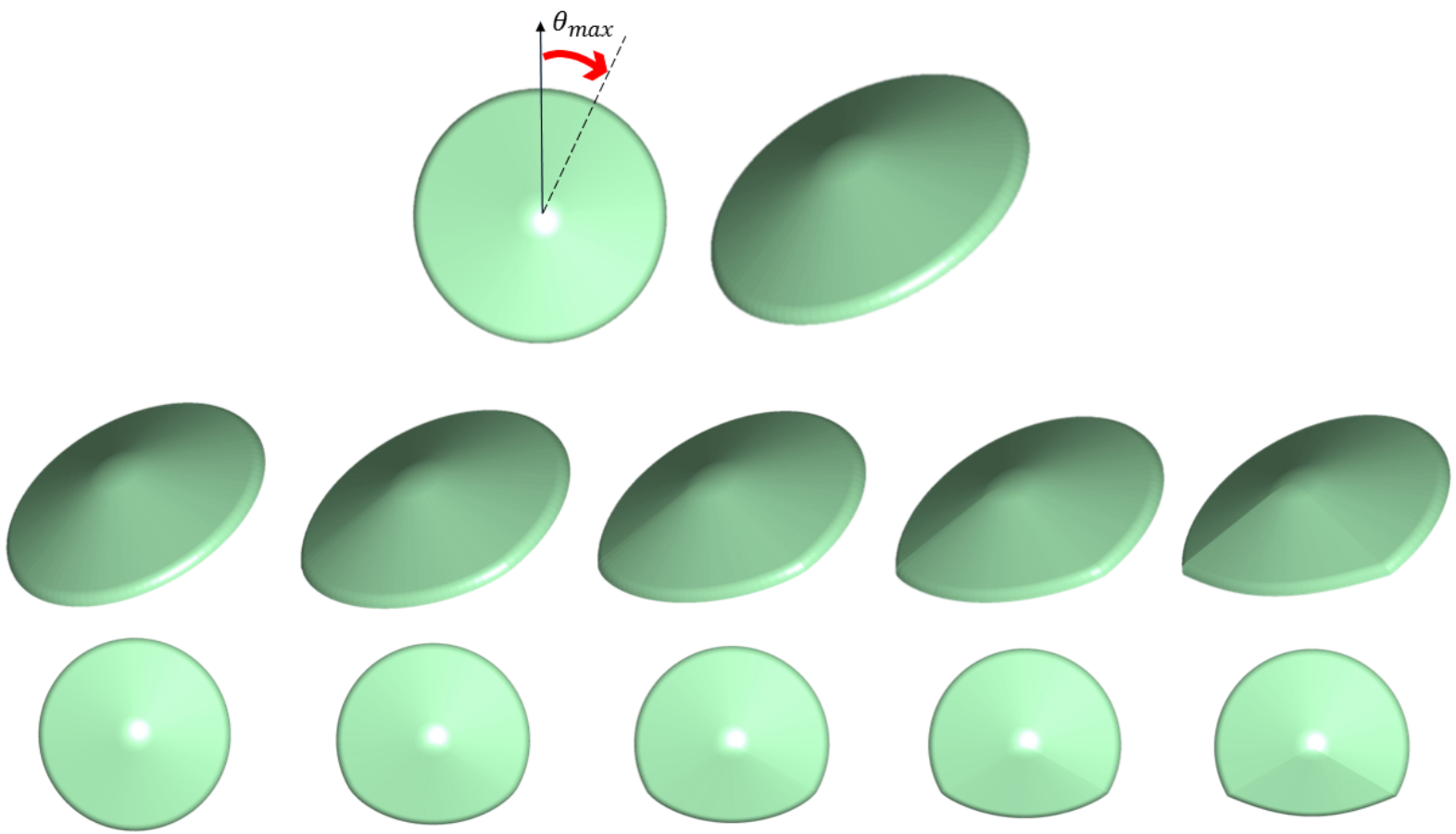

Figure 1: Un-morphed HIAD (top), morphed HIAD at Different Magnitudes (bottom)
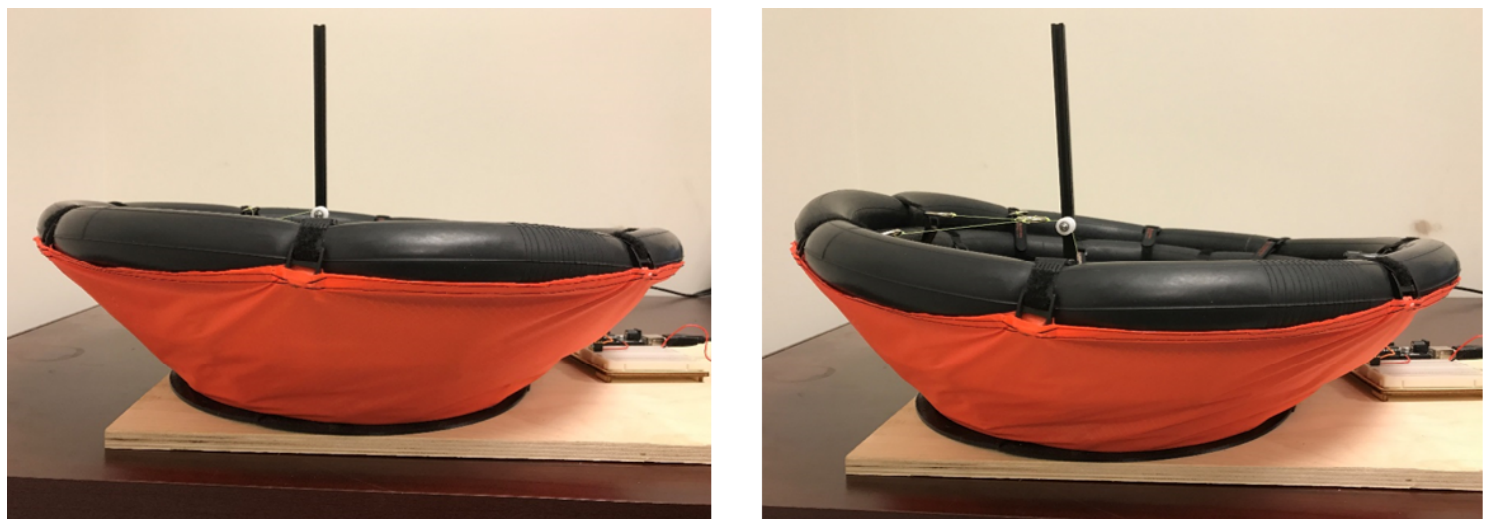

Figure 2: Morphing HIAD Mock Up. Un-morphed (left), Morphed (right)

Atmospheric reentry can be view as a multi-objective optimization problem. The objective is to improve the landing accuracy of the payload while subjecting to constraints such as aero heating and structural loads. Traditionally, this is accomplished primarily with designing an optimized trajectory. The vehicle simply attempts to follow that trajectory through a series of open loop bank angle commands via modulation of the lift vector. With DFC, portions of the HIAD (or trim tabs) could be continuously deformed for down range and cross range corrections which result in more accurate landing footprints. In addition, large ballast mass is not required for offsetting the center of mass. However with the current HIAD/cable configuration (or similar), there may be guidance commands that would cause the one or more of the cables to exceed the limit load. Moreover, it has been shown that peak heating associated with HIADs may not occur necessarily at the stagnation point but at localized areas as the various sections deform. ${ }^{5}$ This motivates the current conceptual-level study that explores the viability of applying an optimal control allocation (OCA) technique to the morphing HIAD configuration. In addition to an optimized trajectory, a well-designed OCA scheme 
could potentially allow a morphing HIAD in real-time to maximize its aerodynamic performance capabilities while keeping the structural loads and aero heating below the respective limits.

The OCA algorithm under investigation is similar to that implemented on NASA Armstrong's Full-Scale Advanced System Testbed ${ }^{6}$ with the exception of an additional term in the cost function that accounts for aero heating. To demonstrate the operation of the OCA, simplified aerodynamics, loads, and aero heating models of a morphing HIAD were developed and implemented into a 3-DOF simulation along with the algorithm itself. A simple guidance scheme was integrated to see how accurate the morphing HIAD along with the OCA can achieve a desired range given loads and aero heating constraints. The rest of the paper is organized as follow: Section II details the mathematics behind the OCA. Section III provides a brief description of the simulation models and OCA operational examples. Section IV provides results from 3-DOF simulation. Section V summarizes the findings and provides discussion for future work.

\section{Optimal Control Allocation Scheme}

The optimal control allocation scheme explored in this study was based on Ref. 6 and modified for the HIAD configuration. Equation (1) shows the cost function, $J$. The cost function is composed of four terms: 1) command tracking, 2) trim, 3) structural loads constraint, 4) aero heating constraint. It was assumed that the loads from the cables are available for feedback via strain gauges. In addition, there are thermocouples located at key locations on the morphing HIAD for heating measurements. The measured structural loads and aero heating vectors, $M$ and $Q$ respectively, must be normalized by the maximum allowable limits such that they do not dominate the cost function.

The command tracking term ensures that the control allocator provides the appropriate surface morphing commands that track the desired commands from the guidance or control system. The trim term attempts to minimize the overall surface deformation (cable retractions). The load constraint cost function term provides a hard constraint that would prevent any cable loads from exceeding the specified limits. The aero heating constraint term provides a similar hard constraint that would prevent any troublesome localized region from exceeding a certain limit. Each term of the cost function is outlined in Eq. (1). Note: The convex nature of the cost function allows for rapid convergence, a characteristic highly desirable for real-time implementation.

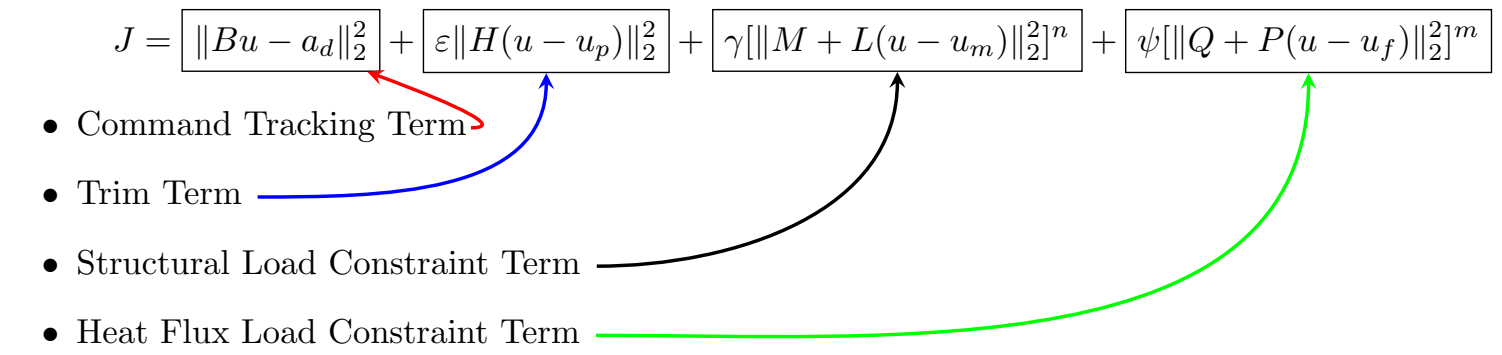

The formulation of the cost function is such that $\partial^{2} J / \partial u^{2}$ is positive definite as long as the $B, H, L$, and $P$ matrices are framed correctly, which results in the solution for $\partial J / \partial u=0$ being a global minimum of the cost function. The Newton - Raphson approach was used to solve $\partial J / \partial u=0$ because of its simplicity, computational efficiency, and desirable convergence properties ${ }^{6}$ and is shown in Eq. (2). Note: the derivation for $\partial J / \partial u$ and $\partial^{2} J / \partial u^{2}$ shown below closely follows the development described in Miller ${ }^{6}$ with the exception of the extra term that accounts for aero heating.

$$
u_{k+1}=u_{k}-R *{\frac{\partial^{2} J^{\dagger}}{\partial u^{2}}}^{\dagger} \frac{\partial J}{\partial u}
$$

The terms from the cost function in Eq. (1) are expanded out in Eq. (3).

$$
\begin{array}{r}
J=\left(B u-a_{d}\right)^{T}\left(B u-a_{d}\right)+\varepsilon\left(u-u_{p}\right)^{T} H^{T} H\left(u-u_{p}\right) \\
+\gamma\left[\left(M+L\left(u-u_{m}\right)\right)^{T}\left(M+L\left(u-u_{m}\right)\right)\right]^{n} \\
+\psi\left[\left(Q+P\left(u-u_{f}\right)\right)^{T}\left(Q+P\left(u-u_{f}\right)\right)\right]^{m}
\end{array}
$$


Equation (3) is simplified and like terms are combined in Eq. (4).

$$
\begin{array}{r}
J=\left[u^{T} B^{T} B u-u^{T} B^{T} a_{d}-a_{d}^{T} B u+a_{d}^{T} a_{d}\right]+\varepsilon\left[u^{T} H^{T} H u-u^{T} H^{T} H u_{p}-u_{p}^{T} H^{T} H u+u_{p}^{T} H^{T} H u_{p}\right] \\
+\gamma\left[M^{T} M+M^{T} L\left(u-u_{m}\right)+\left(u-u_{m}\right)^{T} L^{T} M+\left(u-u_{m}\right)^{T} L^{T} L\left(u-u_{m}\right)\right]^{n} \\
+\psi\left[Q^{T} Q+Q^{T} P\left(u-u_{f}\right)+\left(u-u_{f}\right)^{T} P^{T} Q+\left(u-u_{f}\right)^{T} P^{T} P\left(u-u_{f}\right)\right]^{m}
\end{array}
$$

The first derivative of $J$ with respect to u can be seen in Equation (5).

$$
\begin{array}{r}
\frac{\partial J}{\partial u}=\left[B^{T} B u+\left(B^{T} B\right)^{T} u-B^{T} a_{d}-\left(a_{d}^{T} B\right)^{T}\right]+\varepsilon\left[H^{T} H u+\left(H^{T} H\right)^{T} u-H^{T} H u_{p}-\left(u_{p}^{T} H^{T} H\right)^{T}\right] \\
+\gamma n\left[M^{T} M+M^{T} L\left(u-u_{m}\right)+\left(u-u_{m}\right)^{T} L^{T} M+\left(u-u_{m}\right)^{T} L^{T} L\left(u-u_{m}\right)\right]^{n-1} \\
{\left[\left(L^{T} M\right)^{T}+L^{T} M+L^{T} L\left(u-u_{m}\right)+\left(L^{T} L\right)^{T}\left(u-u_{m}\right)\right]+\psi m\left[Q^{T} Q+Q^{T} P\left(u-u_{f}\right)\right.} \\
\left.+\left(u-u_{f}\right)^{T} P^{T} Q+\left(u-u_{f}\right)^{T} P^{T} P\left(u-u_{f}\right)\right]^{m-1}\left[\left(P^{T} Q\right)^{T}+P^{T} Q^{T} P\left(u-u_{f}\right)+\left(P^{T} P\right)^{T}\left(u-u_{f}\right)\right]
\end{array}
$$

Like terms in Eq. (5) can be combined in Eq. (6) and (7). Equation (8) shows the simplified expression for $\partial J / \partial u$.

$$
\begin{gathered}
N=\left\|M+L\left(u-u_{m}\right)\right\|_{2}^{2} \\
F=\left\|Q+P\left(u-u_{f}\right)\right\|_{2}^{2} \\
\frac{\partial J}{\partial u}=2\left(B^{T}\left[B u-a_{d}\right]+\varepsilon H^{T} H\left(u-u_{p}\right)+\gamma n N^{n-1} L^{T}\left[M+L\left(u-u_{m}\right)\right]+\psi m F^{m-1} L^{T}\left[Q+P\left(u-u_{m}\right)\right]\right)
\end{gathered}
$$

The second derivative of $J$ with respect to $u$ is shown in Eq. (9).

$$
\begin{array}{r}
\frac{\partial^{2} J}{\partial u^{2}}=2\left[B^{T} B+\varepsilon H^{T} H+2 \gamma n(n-1) N^{n-2}\left[L^{T} M+L^{T} L\left(u-u_{m}\right)\right]\left[L^{T} M+L^{T} L\left(u-u_{m}\right)\right]^{T}\right. \\
\left.+\gamma n N^{n-1} L^{T} L+2 \psi m(m-1) F^{m-2}\left[P^{T} Q+P^{T} P\left(u-u_{f}\right)\right]\left[P^{T} Q+P^{T} P\left(u-u_{f}\right)\right]^{T}+\gamma m F^{m-1} P^{T} P\right]
\end{array}
$$

Tuning of the parameters in the control allocator is an important step to ensure that the cost function is minimized while achieving good convergence properties and reasonable surface deflection commands. This was done on a trial and error basis and closely follows the procedures outlined in Ref. 6 .

\section{HIAD Models and OCA Operation}

Simplified models were used to demonstrate the feasibility of this optimal control allocation scheme on a morphing HIAD. The key assumptions made in these models are listed below:

- Loads measurement are available for each cable and represents the force within the cable

- Structural (cable) load increases linearly with cable retraction

- Aero heating model is a function of atmospheric properties and cable retraction

- The HIAD can maintain a smooth (second order continuous) shape when morphed

\section{HIAD Configuration}

The morphing HIAD has four separation morphing sections shown in Fig. 3. Sections 1 and 3 provide longitudinal (down range) control of the HIAD, while sections 2 and 4 provide lateral (cross range) control. Each morphing section has 7 cables to alter the shape of the HIAD and is shown in Fig. 4. There are certain constraints on the possible morphed shapes for each section. ${ }^{4}$ 


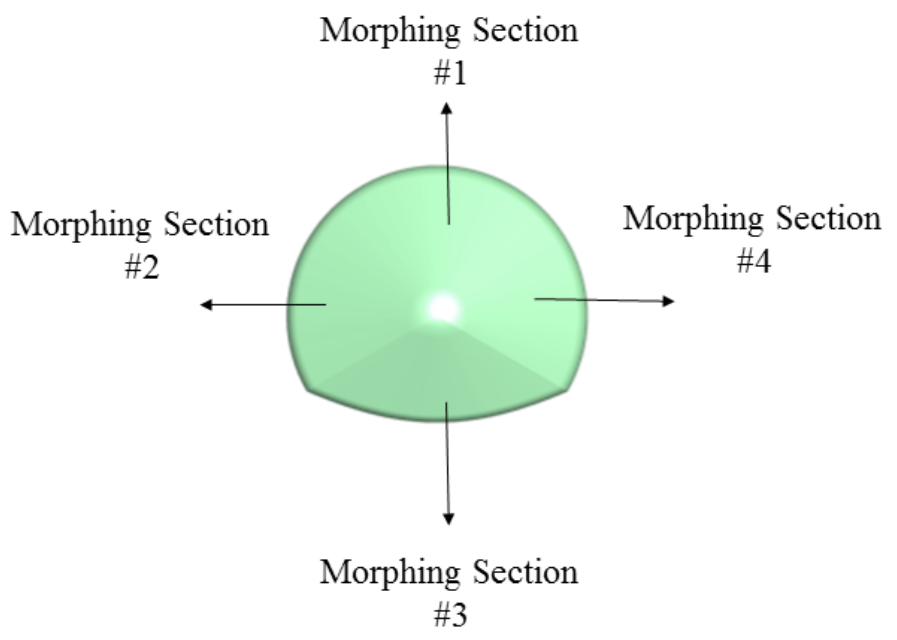

Figure 3: Morphed HIAD Example: Section 3 Morphed

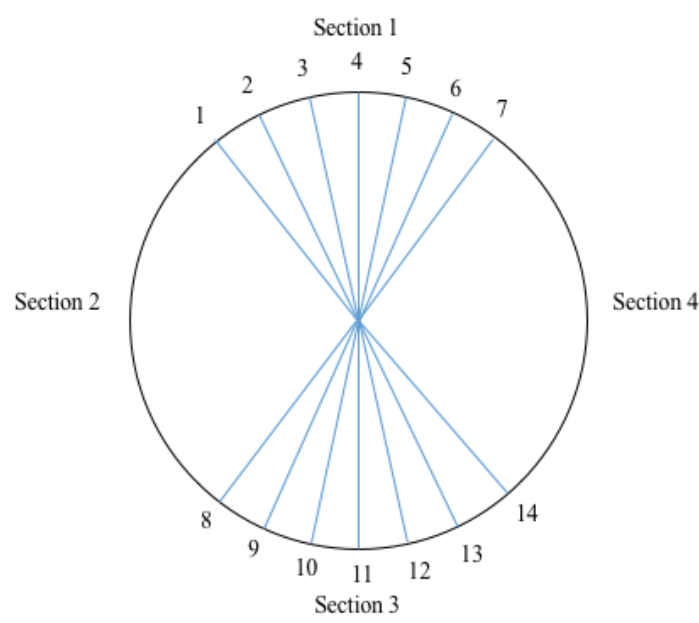

Figure 4: Cable Numbering

\section{Structural Model}

A linear loads model that predicts the cable loads with displacement was used in the simulation. The loads model for each case can be seen in Table 1. $n$ represents the cable number, $l_{n}$ represents the load of each cable (function of cable displacement), $u_{n}$ represents the cable displacement, $l_{\text {max }}$ represents the maximum allowable cable load (based on material constants), $u_{\min }$ and $u_{\max }$ represents the cable retraction limits. $u_{\max }$ is the greatest for the center cable and gradually tapers off towards the edges of the morphing section. $l_{n}$ and $u_{\max }$ are taylored to enforce a quadratic deformed shape.

\begin{tabular}{|c|c|c|c|c|c|}
\hline Section Number & Cable Number $(n)$ & $l_{n}(N)$ & $l_{\max }(N)$ & $u_{\min }(m)$ & $u_{\max }(m)$ \\
\hline \multirow{5}{*}{1} & 1 & $3.509 e 3 \times u_{1}$ & 1754.5 & 0.0 & 0.50 \\
& 2 & $2.287 e 3 \times u_{2}$ & 1754.5 & 0.0 & 0.77 \\
& 3 & $1.807 e 3 \times u_{3}$ & 1754.5 & 0.0 & 0.97 \\
& 4 & $1.141 e 3 \times u_{4}$ & 1754.5 & 0.0 & 1.54 \\
& 5 & $1.807 e 3 \times u_{5}$ & 1754.5 & 0.0 & 0.97 \\
& 6 & $2.287 e 3 \times u_{6}$ & 1754.5 & 0.0 & 0.77 \\
& 7 & $3.509 e 3 \times u_{7}$ & 1754.5 & 0.0 & 0.50 \\
\hline \multirow{5}{*}{3} & 8 & $3.509 e 3 \times u_{8}$ & 1754.5 & 0.0 & 0.50 \\
& 9 & $2.287 e 3 \times u_{9}$ & 1754.5 & 0.0 & 0.77 \\
& 10 & $1.807 e 3 \times u_{10}$ & 1754.5 & 0.0 & 0.97 \\
& 11 & $1.141 e 3 \times u_{11}$ & 1754.5 & 0.0 & 1.54 \\
& 12 & $1.807 e 3 \times u_{12}$ & 1754.5 & 0.0 & 0.97 \\
& 13 & $2.287 e 3 \times u_{13}$ & 1754.5 & 0.0 & 0.77 \\
& 14 & $3.509 e 3 \times u_{14}$ & 1754.5 & 0.0 & 0.50 \\
\hline
\end{tabular}

Table 1: Structural Loads Model

\section{Aerodynamic Model}

Aerodynamic data was generated for a HIAD with four morphing sections based on CBAERO. ${ }^{7}$ As shown in Fig. 3, deformation of sections 1 and 3 generate lift, while sections 2 and 4 generate side force. The aerodynamic model for sections 1 and 3 is a function of incremental change in $\mathrm{L} / \mathrm{D}(\delta(\mathrm{L} / \mathrm{D}))$ versus individual cable retraction. The assumption is that each cable provides some contribution to the total $(\Delta(\mathrm{L} / \mathrm{D}))$ for 
each morphing section. The control effectiveness matrix per morphing section is shown in Eq. (10). Here $n$ is the number of cables in a given section.

$$
B=\left[\begin{array}{lllll}
\frac{d\left(C_{L} / C_{D}\right)}{d \delta_{1}} & \frac{d\left(C_{L} / C_{D}\right)}{d \delta_{2}} & \ldots & \frac{d\left(C_{L} / C_{D}\right)}{d \delta_{n-1}} & \frac{d\left(C_{L} / C_{D}\right)}{d \delta_{n}}
\end{array}\right]
$$

\section{Heat Flux Model}

It has been shown that the region of peak heating on a morphing HIAD may not necessarily occur at the stagnation pressure ${ }^{5}$ but could occur near the shoulder portions as the HIAD deforms. For the purpose of demonstrating how the OCA handles the additional aero heating constraint, a simple heating model as a function of surface morphing (cable retractions) was developed. The model was based on Ref. 8 and is shown in Eqs. (11) and (12). Equation (11) is the heat flux on the entire HIAD, while Eq. (12) represents the localized heat flux that is a function of individual cable retraction, $u_{n}$. Here $U_{\infty}$ is the free-stream velocity, $\rho$ is the free-stream density, $r_{n o s e}$ is the radius of the HIAD's nose. The maximum allowable heat flux per cable is $20,000 \mathrm{~W} / \mathrm{m}^{2} .{ }^{9}$ Note: the current model is not an accurate representation of the actual heating one would measure on a HIAD due to surface morphing (function of many variables such as angle-of-attack, angle-of-sideslip, Mach number, various surface deformations, etc).

$$
\begin{gathered}
\dot{q}=(0.000183) U_{\propto}^{3} \sqrt{\frac{\rho}{r_{\text {nose }}}} \\
\dot{q_{n}}=\frac{1}{20} \dot{q}\left|u_{n}\right|
\end{gathered}
$$

\section{Optimal Control Allocator Block}

Figure 5 shows the top level block diagram of the OCA. The inputs to the OCA include the control effectiveness matrix that is scheduled with flight condition, the L/D command, and measurements from the loads and aero heating models. The outputs of the OCA are cable retractions and RCS thrust commands. Note: the RCS thrusters are only active in the event that the morph surfaces are unable to achieve the L/D command (due to constraints) and not the focus of this study.

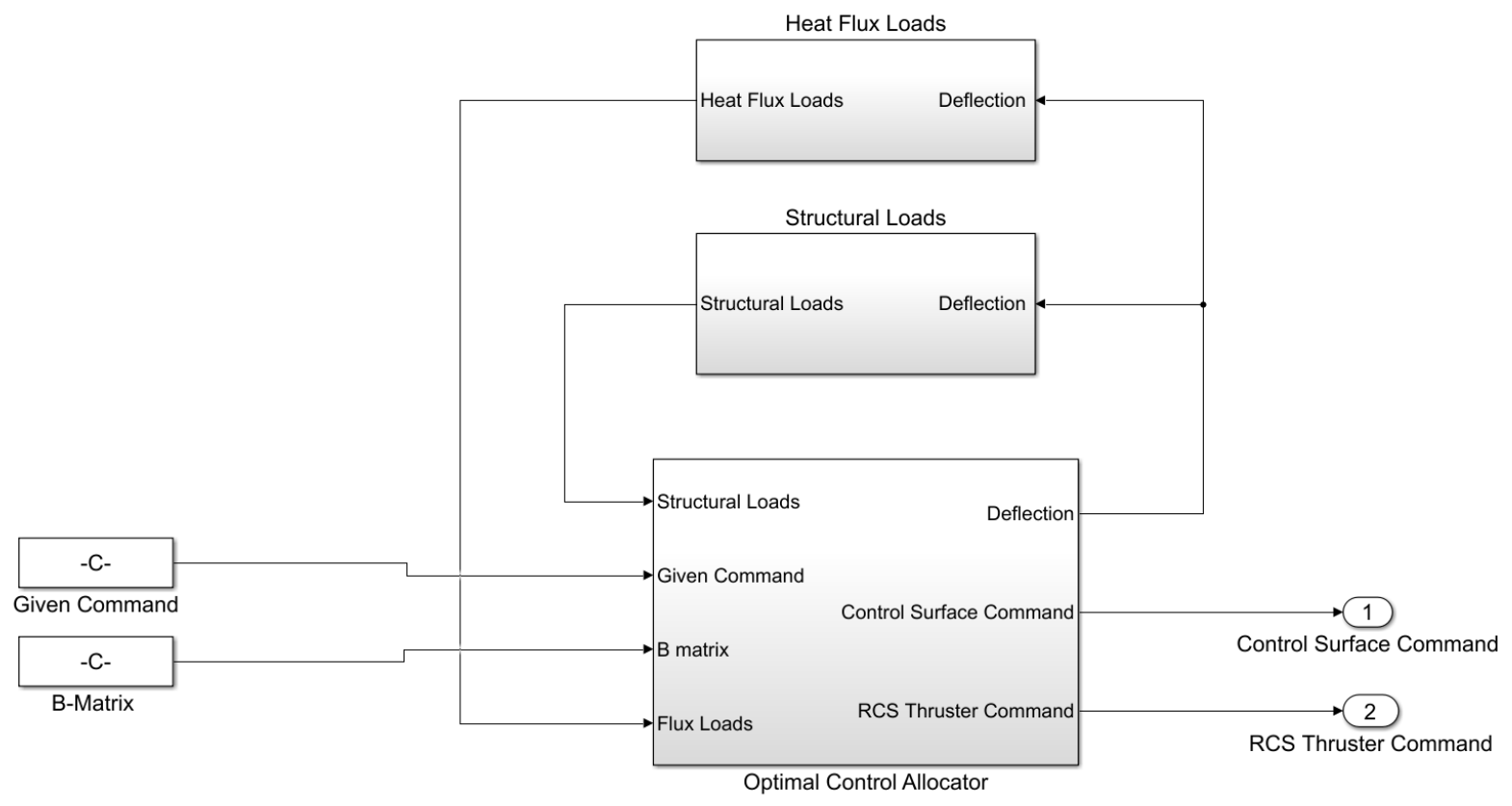

Figure 5: OCA Block 


\section{OCA Operational Examples and Results}

Figures 6 - 8 show examples of how the OCA performs under various operational scenarios for a discrete morphing event. Three cases shown are: 1) nominal operation, acceptable loads and aero heating measurements. 2) structural load limit exceeded. 3) aero heating limit exceeded. These three cases are tabulated in Table 2. From $\mathrm{T}=0$ to 100 seconds, the HIAD is undeformed and assume to have a (L/D)o of 0.0505 due to $\mathrm{CG}$ shift. At $\mathrm{T}=100$ seconds, a discrete $\mathrm{L} / \mathrm{D}$ command is issued.

\begin{tabular}{|c|c|c|c|}
\hline Morphing Time & Acceptable $(\mathrm{L} / \mathrm{D})_{c}$ & Unacceptable Structural $(\mathrm{L} / \mathrm{D})_{c}$ & Unacceptable Aerothermal $(\mathrm{L} / \mathrm{D})_{c}$ \\
\hline 100 & 0.0547 & 0.216 & 0.136 \\
\hline
\end{tabular}

Table 2: Acceptable and Unacceptable L/D commands for HIAD

Figure 6 shows results for case 1 . To produce the $\mathrm{L} / \mathrm{D}$ command at $\mathrm{T}=100 \mathrm{sec}$, section 3 was deformed. The loads and heating remained below their respective limits after the morphing event. Time histories of the cable retractions, structural loads, control commands, and aero heating are shown.
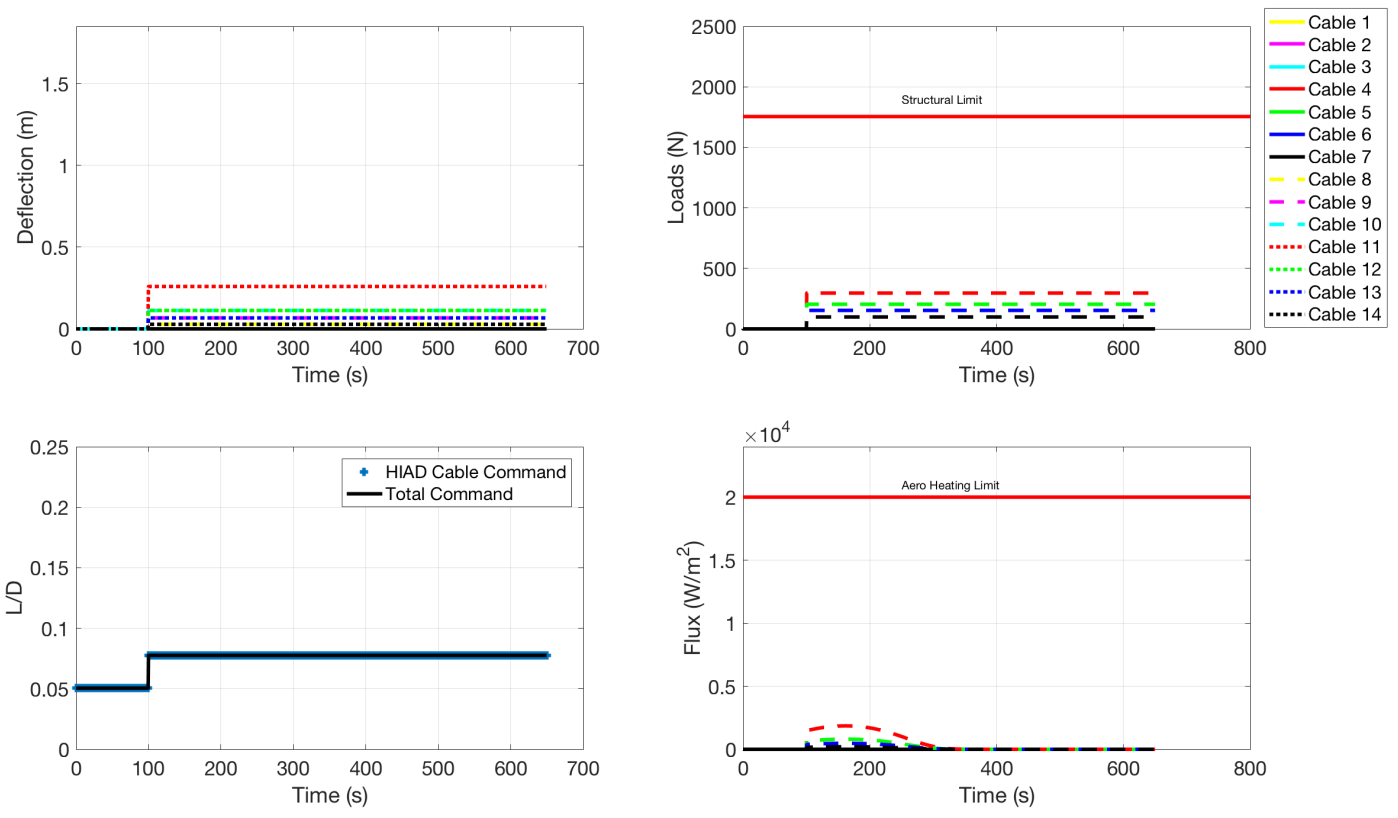

Figure 6: Acceptable Loads and Aero Heating

Figure 7 shows results for case 2 . The $\mathrm{L} / \mathrm{D}$ command at $\mathrm{T}=100$ seconds yield an initial morphing solution that caused Cable 11 to exceed the maximum allowable structural load of $1754.7 \mathrm{~N}$. The structural and heating measurements were assumed to be available to the OCA in real-time and the algorithm immediately recognized a violation in the cable loads. OCA subsequently converged on a new solution by reducing the retraction for Cable 11 and increasing retractions for the neighboring cables. Hence, the system was still able to achieve the L/D command. 

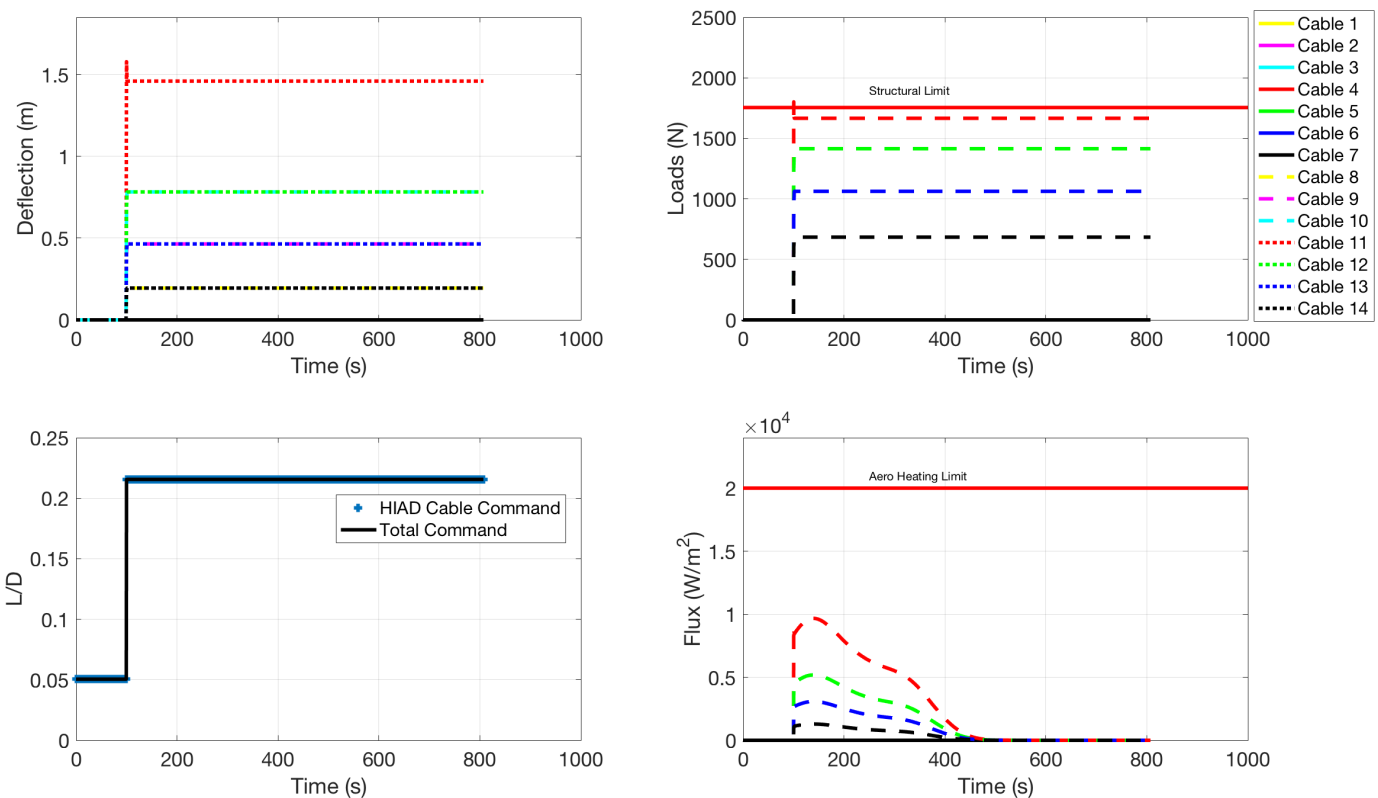

Figure 7: Unacceptable Loads

Figure 8 shows results for case 3 in which the L/D command resulted in an initial morphing solution that caused the aero heating limit to be exceeded. Consequently from $\mathrm{T}=100$ to 200 seconds, the OCA converged to a solution where the Cable 11 retraction command was reduced from the initial solution. Beyond $\mathrm{T}=$ 200 seconds, the vehicle exited the region of maximum aero heating and the OCA settled on a new solution that minimized overall cable activities (by increasing retraction for Cable 11 and decreasing retractions for the neighboring cables).
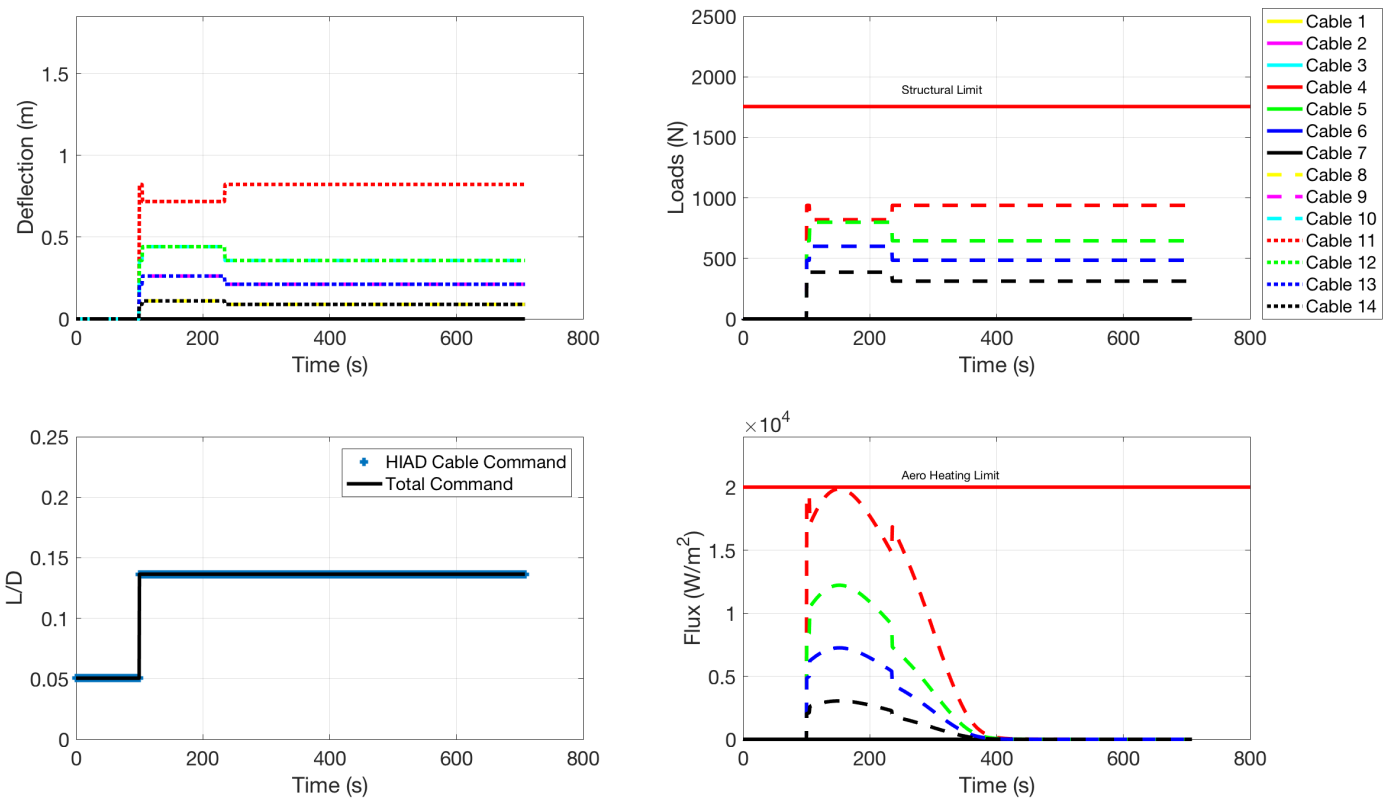

Figure 8: Unacceptable Aero Heating 


\section{Trajectory Simulation}

\section{Overview}

The OCA along with the models described in Section III were integrated into a 3DOF trajectory simulation. ${ }^{1}$ It assumes an entry into a rotating and spherical Earth with the atmosphere rotating with the Earth and no winds. The atmospheric density, temperature, and viscosity are assumed to depend only on altitude. Two studies on the performance of the OCA were investigated. The first consisted of a series of discrete morphing events that requested changes in L/D at specified times during the trajectory. The second integrated a variable gain guidance scheme into the simulation that updates the $\mathrm{L} / \mathrm{D}$ command at each time-step. Here the guidance scheme is attempting to track a pre-determined trajectory.

\section{Multiple Discrete Morphing Events Results}

Initial conditions (based off the IRVE-3 flight test mission) for the multiple discrete morphing simulation are shown in Table 3. The discrete L/D commands are shown in Table 4. It was assumed that the undeformed HIAD has a baseline L/D of 0.0505 . Hence from $\mathrm{T}=0$ to 100 seconds, no morphing commands were issued.

\begin{tabular}{|c|c|}
\hline$\gamma($ deg $)$ & $-1.3^{\circ}$ \\
\hline$h_{o}(m)$ & 89982 \\
\hline$v_{e}(m / s)$ & 8054.3 \\
\hline
\end{tabular}

Table 3: Initial Conditions for Trajectory Simulation with Multiple Discrete Morphing Events

\begin{tabular}{|c|c|}
\hline Morphed Time (s) & L/D Command \\
\hline 0 & 0.0505 \\
\hline 100 & 0.0799 \\
\hline 150 & 0.2122 \\
\hline
\end{tabular}

Table 4: L/D commands

Figure 9 shows the control allocation results. These are similar to those shown in Section III. It can be seen that at $\mathrm{T}=150$ seconds the structural load limit was exceeded in Cable 11 as a result of the inital morphing solution. The OCA sensed the loads constraint was violated and converged to a new solution. As a result, Cables 8, 9, 10,12,13, and 14 increased their retractions to compensate for Cable 11 while perfect command tracking was maintained. 

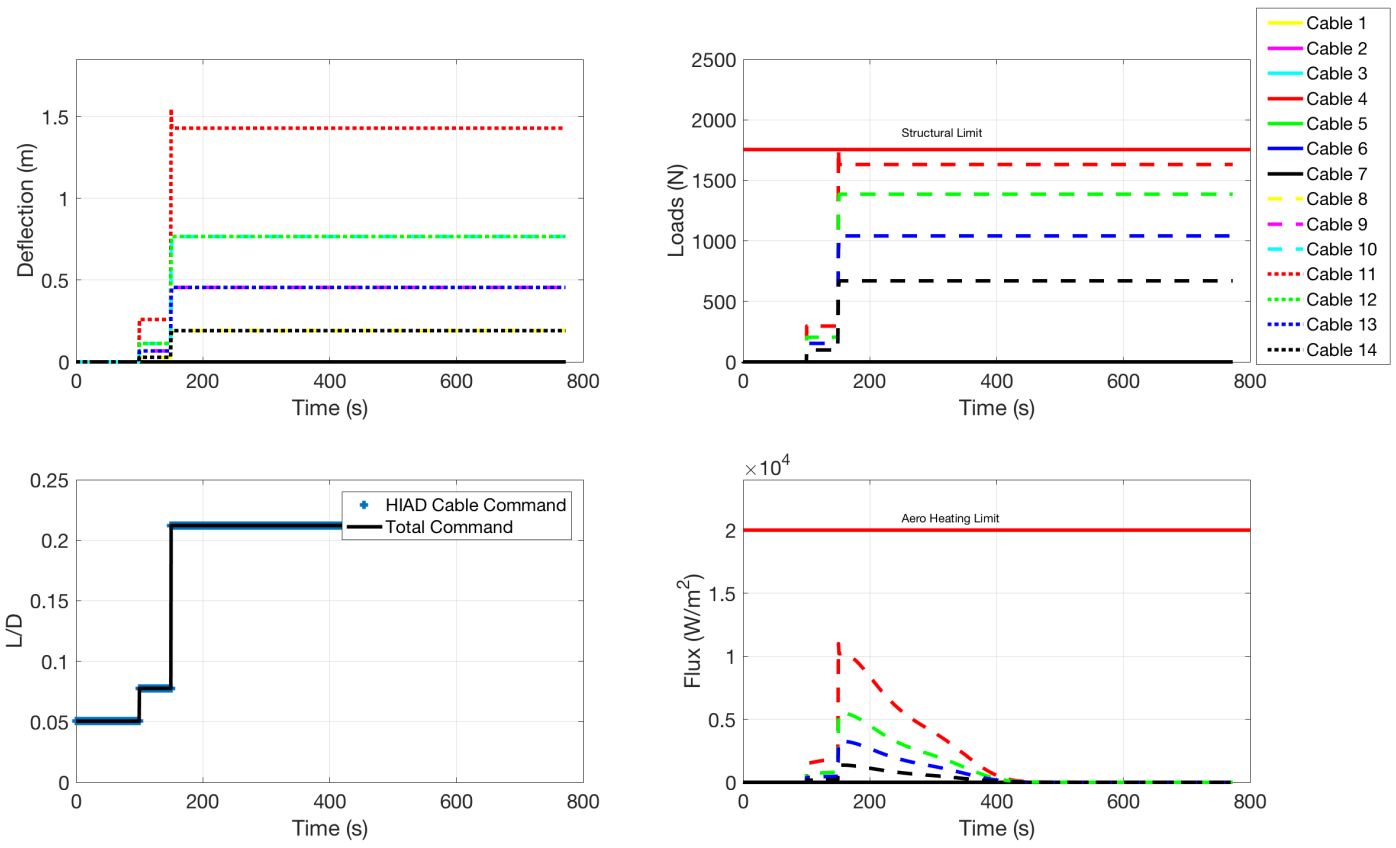

Figure 9: Optimal Control Allocation Results for Multiple Discrete Morphing Events

Figures 10 and 11 show the time histories of altitude and down range distance. The altitude versus time and free-stream velocity is shown in Fig. 10. The two discrete morphing events are represented by the black lines. It is apparent that after the second morphing event, the HIAD is able to stay aloft a lot longer compare to the baseline (undeformed) case as expected. This results to an increase in down range capabilities as shown in Fig. 11.
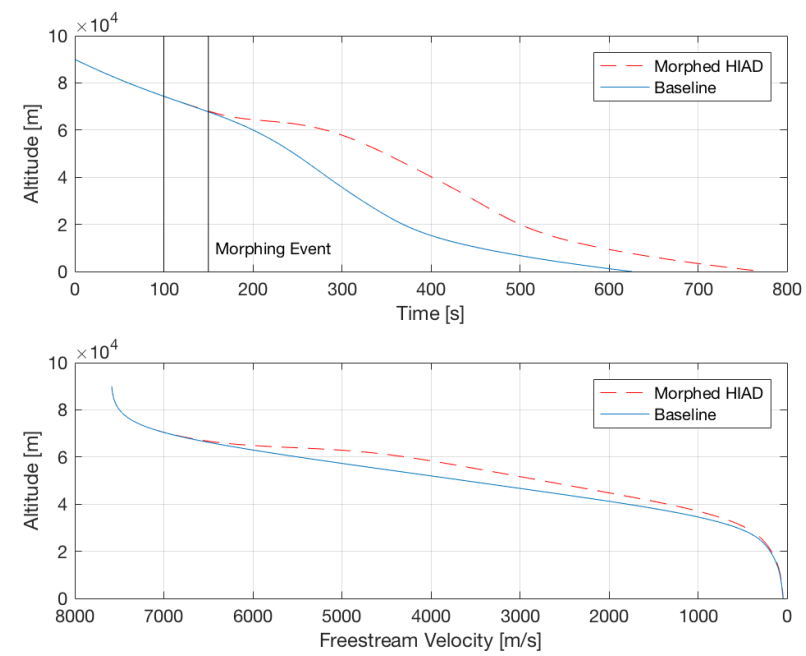

Figure 10: Altitude Time History: Multiple Discrete Morphing vs. Baseline 


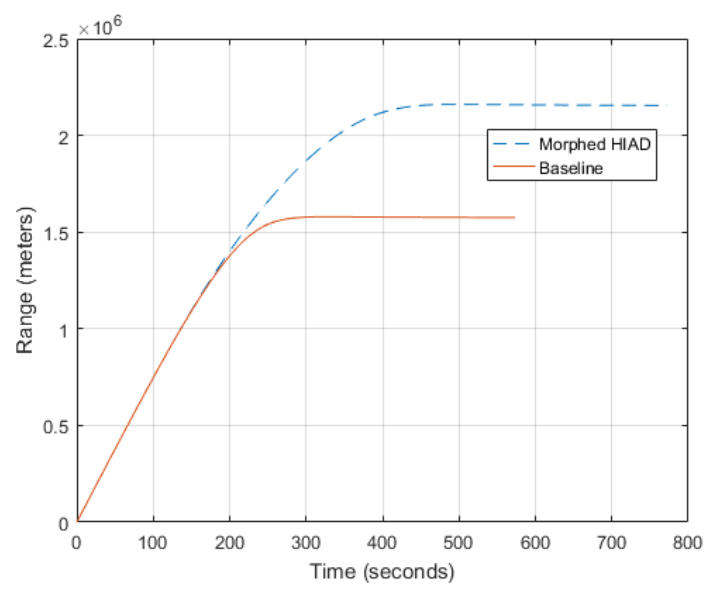

Figure 11: Range Time History: Multiple Discrete Morphing vs. Baseline

The heat flux versus time and free-stream velocity is shown in Fig. 12. It is apparent from the heat flux versus free-stream velocity subplot that morphing trajectory experienced lower peaking heat compare to the baseline case. This illustrates another potential benefit of the morphing HIAD in its capability to control the peaking heating by lofting the trajectory in real-time.
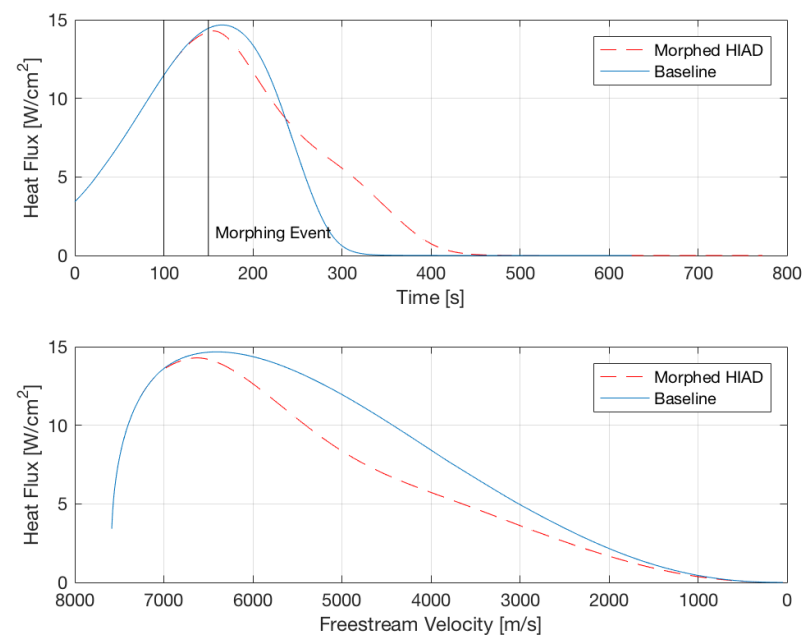

Figure 12: Aero Heating Time History: Multiple Discrete Morphing vs. Baseline

\section{Results with Variable L/D Morphing Events}

A variable-gain guidance scheme was implemented into the trajectory simulation for the morphing HIAD to track a desired trajectory by continuously updating the $\mathrm{L} / \mathrm{D}$ commands in real-time. The scheme shown in Eq. (13) is an implicit guidance law using a reference trajectory and based on that implemented on the OREX mission. ${ }^{10}$ The guidance was updated every 5 seconds throughout the trajectory. The accuracy deteriorates if the initial conditions between the reference trajectory and actual flight trajectory becomes too large. In the simulation it was assumed that perfect estimates of flight path angle $(\gamma)$, drag (D), and $\mathrm{R}$ (range to go) were available at each time step. $K_{1}, K_{2}$, and $K_{3}$ were tuned to ensure a plausible L/D command at each guidance update.

$$
\left(\frac{L}{D}\right)_{c}=\left(\frac{L}{D}\right)_{r e f}+K_{1}\left(\gamma-\gamma_{r e f}\right)+K_{2}\left[\left(\frac{D}{W}\right)-\left(\frac{D}{W}\right)_{r e f}\right]+K_{3}\left(R-R_{r e f}\right)
$$

Table 5 displays the initial conditions for the reference and perturbed trajectories respectively. For this case, the differences in the initial conditions between the two trajectories were too large which led to a $25.5 \%$ 
error in the final down range distance as shown in Table 6.

\begin{tabular}{|c|c|c|}
\hline Initial Condition & Reference Trajectory & Off-Nominal Trajectory \\
\hline$C_{L} / C_{D}$ & 0.0505 & 0.1067 \\
\hline$\gamma($ deg $)$ & $-1.3^{\circ}$ & $-1.4^{\circ}$ \\
\hline$h_{0}(m)$ & 89982 & 89000 \\
\hline$v_{e}(m / s)$ & 8054.3 & 8060 \\
\hline
\end{tabular}

Table 5: Initial Conditions

\begin{tabular}{|c|c|c|}
\hline Desired Range $(\mathrm{m})$ & Actual Range $(\mathrm{m})$ & Percent Difference \\
\hline $1.95 \mathrm{e} 6$ & $1.45 \mathrm{e} 6$ & 25.5 \\
\hline
\end{tabular}

Table 6: Comparison of Reference and Actual Range

Figure 13 shows the time histories of the OCA parameters as it attempted to track the time varying L/D commands from the guidance scheme. Note: for this example the structural load limit was reduced by a factor of 8 from that shown in Table 1 to properly demonstrate the operational scenario of the OCA in the event of excessive cable loads. At approximately $\mathrm{T}=200$ seconds, the allocation solution caused the Cable 11 load reach the maximum allowable. The OCA sensed a violation in the structural loads and converged to a new solution. As a result, Cables 8, 9, 10, 12, 13, and 14 increased their retractions to compensate for Cable 11 while perfect command tracking was maintained.
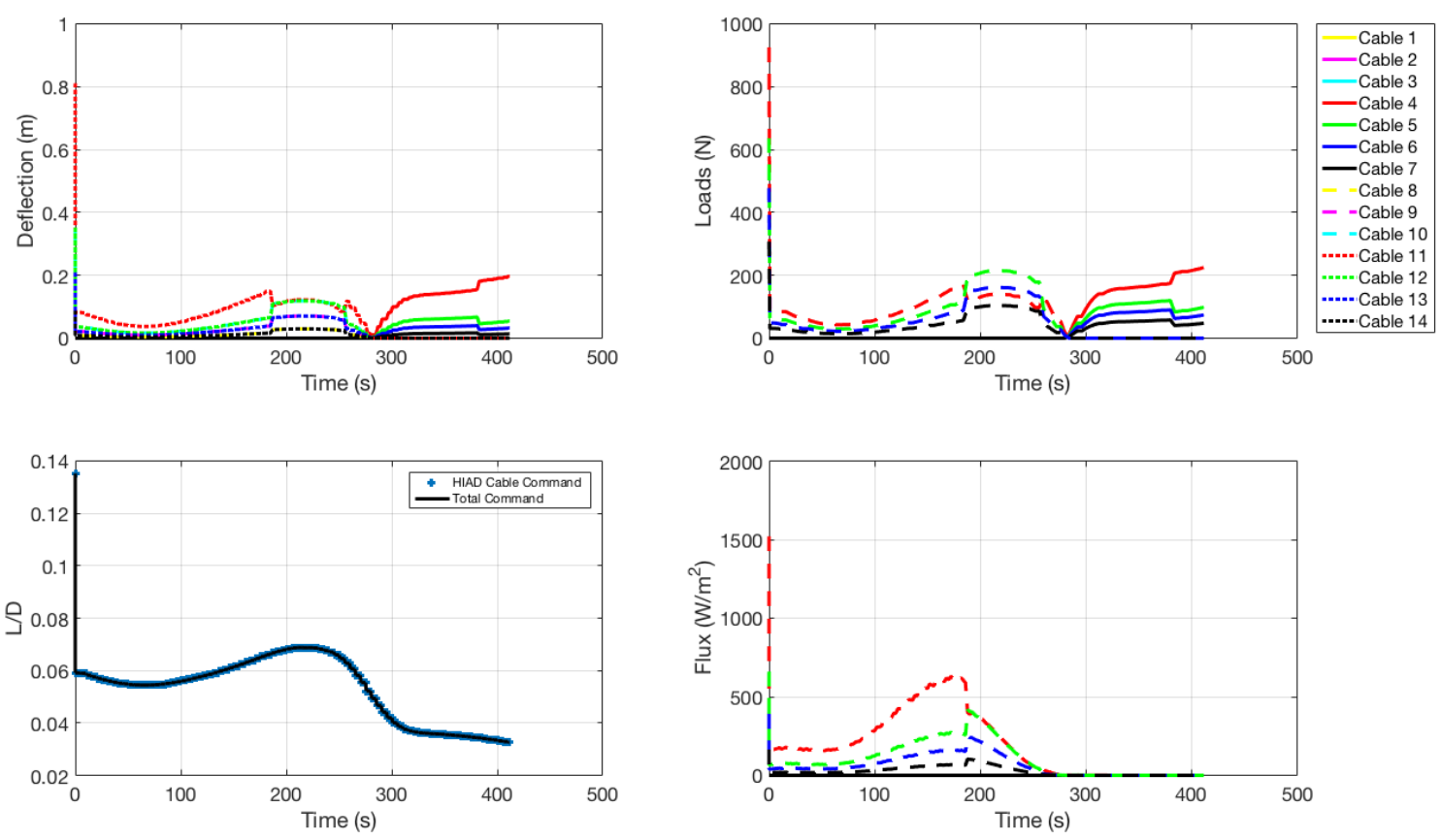

Figure 13: Optimal Control Allocation Results for Variable Morphing Case

Figure 14 shows the altitude versus time and free-stream velocity time histories. The variable gain guidance scheme along with OCA does a reasonable job of tracking the reference trajectory despite the large differences in the initial conditions and loads/aero heating constraints. Simulation was terminated at around 400 seconds as the system reached the altitude trigger. 

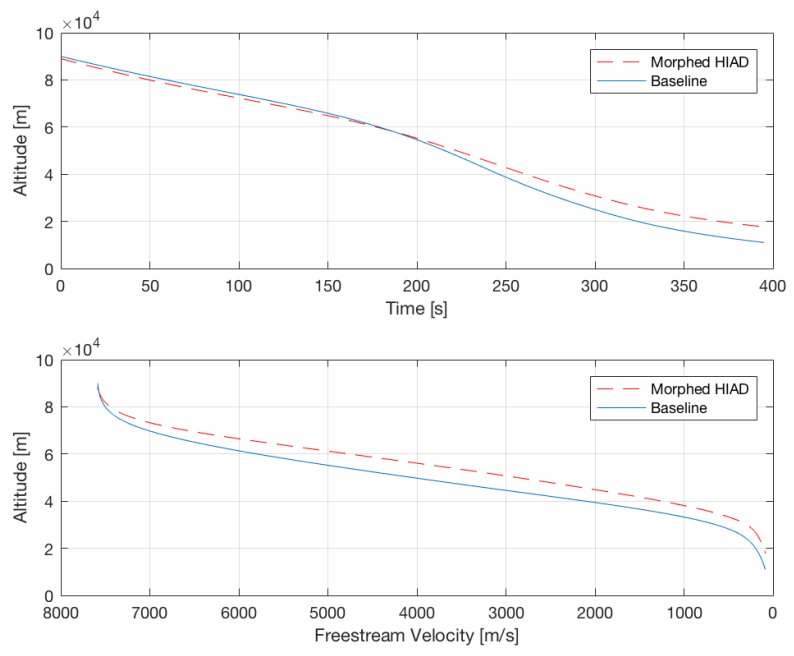

Figure 14: Altitude Time History: Variable Morphing Case

\section{Conclusion}

With the need for precision landing of large payloads on Mars, the morphing HIAD concept offers potentially a significant improvement in landing footprint accuracy compare to the conventional bank angle control approach. Other benefits include packaging, scalability, and need for large ballast. However, with the ability to modulate its shape for continuous down range and cross range control, there may be cases in which the morphing commands causes certain structural loads or aero heating limits be exceeded. The benefits of implementing an optimal control allocation (OCA) algorithm on a morphing HIAD was demonstrated in this paper. The OCA can allow the morphing HIAD in real-time to maximize its aerodynamic performance capabilities while keeping structural loads and aero heating below their respective limits. The simple architecture allows the designer to readily incorporate additional system constraints and its convex nature is highly desirable for real-time implementation.

\section{Acknowledgments}

The authors would like to thank everybody who provided feedbacks during the process of writing this paper. Specifically Dr. Jerry Newsom and Steve Derry for providing the initial aerodynamic database, the HIAD team at NASA Langley for feedbacks on the HIAD configuration and EDL concept of operations, and Susan Frost of NASA Ames and Chris Miller of NASA Armstrong for feedbacks on the optimal control allocation scheme. The first author would like to thank Dr. Elizabeth Ward for the opportunity to work at NASA as an intern. 


\section{References}

${ }^{1}$ Green, J., Lindberg, R., and Dunn, B., "Morphing Hypersonic Inflatable Aerodynamic Decelerator," AIAA, 2013.

${ }^{2}$ Hughes, S. and Cheatwood, F. M., "Hypersonic Inflatable Aerodynamic Decelerator (HIAD) Technology Development Overview," AIAA, 2011.

${ }^{3}$ Cianciolo, A. and Powell, R. W., "Entry, Descent, and Landing Guidance and Control Approaches to Satisfy Mars Human Mission Landing Criteria," AAS, 2017.

${ }^{4}$ Slagle, A. M., "Morphing HIAD Structural Loads Data," .

${ }^{5}$ Hollis, B. R., "Boundary-Layer Transition and Surface Heating Measurements on a Hypersonic Inflatable Aerodynamic Decelerator with Simulated Flexible TPS," AIAA, 2017.

${ }^{6}$ Miller, C. J. and Goodrick, D., "Optimal Control Allocation with Load Sensor Feedback for Active Load Suppression, Experiment Development," AIAA, 2017.

7 "Configuration-Based Aerodynamics by NASA Ames," .

${ }^{8}$ FAA, "Returning from Space: Re-entry," .

${ }^{9}$ Tauber, M. E. and Meenes, G. P., "Aerothermodynamics of Transatmospheric Vehicles," AIAA, 1987.

${ }^{10}$ Matsumoto, S., Kondoh, Y., Suzuki, Y., Imada, T., Sato, N., Yamamoto, H., Kobayashi, S., and Motoyama, N., "Accurate Real-Time Prediction Guidance Using Numerical Integration for Reentry Spacecraft," AIAA, 2013. 